Commun. Korean Math. Soc. 26 (2011), No. 4, pp. 709-720

http://dx.doi.org/10.4134/CKMS.2011.26.4.709

\title{
NUMERICAL SOLUTION OF STOCHASTIC DIFFERENTIAL EQUATION CORRESPONDING TO CONTINUOUS DISTRIBUTIONS
}

\author{
Mohammad Amini, Ali Reza Soheili, and Mahdi Allahdadi \\ ABstract. We obtain special type of differential equations which their \\ solution are random variable with known continuous density function. \\ Stochastic differential equations (SDE) of continuous distributions are de- \\ termined by the Fokker-Planck theorem. We approximate solution of dif- \\ ferential equation with numerical methods such as: the Euler-Maruyama \\ and ten stages explicit Runge-Kutta method, and analysis error predic- \\ tion statistically. Numerical results, show the performance of the Rung- \\ Kutta method with respect to the Euler-Maruyama. The exponential \\ two parameters, exponential, normal, uniform, beta, gamma and Parreto \\ distributions are considered in this paper.
}

\section{Introduction and preliminaries}

Many physical systems are modeled by ordering differential equations. In many models for describing physical phenomena, stochastic terms were omitted because there are not powerful numerical methods and high performance computers. Some applications of stochastic differential equations are investment funds, population dynamic, polymer dynamic, Sntyk protein and genetic science. Also stochastic concepts apply in chemical movement models and physic. History of appearance of stochastic differential equation has been a time to solve different physical and mathematical problems including random variables and quantities. Despite that in many cases, ignoring stochastic terms in some phenomena lead to simpler untrue analysis. As a result, stochastic quantities were considered for obtaining actual models. Many authors have been investigated the numerical solution of stochastic differential equations (see for example, Mao and Sabanis ([16]), Buckwar ([3]), Tocino and Ardanuy ([23]), Mao et al. ([17]), Kim and Stanescu ([12]) and Carletti ([6])). Moreover Burrage et al. ([4]) has been studied a review of recent progress in the design of numerical methods for computing sample paths of solutions to stochastic differential equations.

Received July 17, 2010; Revised November 16, 2010.

2010 Mathematics Subject Classification. Primary 65C20; Secondary 65C10, 65C30.

Key words and phrases. stochastic differential equation, continuous distribution function, confidence interval, Euler-Maruyama method. 
In this paper, stochastic differential equations were considered where their solution is a random variable with known distribution, this stochastic differential equations (SDE) of continuous distributions are determined by the FokkerPlanck theorem, special distributions like uniform, exponential (with one and two parameters), normal, beta, gamma and Parreto were studied. Before presenting the main results, we need to review some definitions and theorems. In Section 2, the Euler-Maruyama as a method for approximating stochastic differential equations and its error estimation and also confidence interval for the error will be presented. Section 3 shows how to determine differential equations of the continuous distributions and finally in Section 4, we approximate the solution with a Runge-Kutta method.

Definition 1.1. Let $\{X(t), t \geq 0\}$ be a real valued Markov process, the transition distribution function is defined

$$
F(y, s, x, t)=P[X(t) \leq x \mid X(s)=y],
$$

where $x, y \in R$ and $0<s<t$. Density function (if there exist) is obtained

$$
f(y, s, x, t)=\frac{\partial F(y, s, x, t)}{\partial x} .
$$

Definition 1.2. Let $\{X(t), t \geq 0\}$ be a stochastic process with real valued, where for $F \in L^{1}(0, T), G \in L^{2}(0, T)$ and all times $0 \leq s \leq r \leq T$, satisfy in

$$
X(r)=X(s)+\int_{s}^{r} F d t+\int_{s}^{r} G d W
$$

then $X(t)$ for $0 \leq t \leq T$ has the Ito stochastic differential equation $d X=$ $F d t+G d W$, where $L^{i}(0, T), i=1,2$ are the space of all real stochastic processes and measurable $F$ such that $E\left(\int_{0}^{T}|F|^{i} d t\right)<\infty$.

Theorem 1.1 (Burrage, [5]). The Ito stochastic differential equation $d X=$ $a(t, X) d t+b(t, X) d W$ has an unique solution, if $a(t, X)$ and $b(t, X)$ satisfy in the Lipschitz conditions and Linear-growth bounded condition.

Let $\{X(t), t \geq 0\}$ be a diffusion stochastic process and $\frac{\partial F(y, s, x, t)}{\partial y}, \frac{\partial^{2} F(y, s, x, t)}{\partial y^{2}}$ exist and continuous in each components. Then the transition density function $f(y, s, x, t)$ satisfies in the following partial differential equation,

$$
\frac{\partial f(x, t)}{\partial t}=-\frac{\partial(a(x, t) f(x, t))}{\partial x}+\frac{1}{2} \frac{\partial^{2}\left(b^{2}(x, t) f(x, t)\right)}{\partial x^{2}},
$$

this equation is known as the Kolmogorov's forward equation (Kloeden and Platen $([13]))$.

The Fokker-Planck equation in the form (1.1) was obtained to study Brownian motion and is sometimes called a diffusion equation. The coefficient $a(x, t)$ describes the average value of the particle displacement and is also known as the drift. Likewise, $b(x, t)$ can be interpreted as a variance of a particle's displacement from the average value and is called the diffusion coefficient (For more 
details see Primak et al. ([20]) and Risken ([21])). Moreover, many authors studied the Fokker-Planck equations and its applications, such as; Langtangen ([15]), Paola and Sofi ([19]), Ditlevsen ([9]), Chen and Li ([7]), Frank ([11]), Ambrosio et al. ([1]), Barkai ([2]), Chow and Zhou ([8]) Torres and Figueiredo ([22]) and Frank and Daffertshofer ([10]). Ottinger ([18]) proved that if density function satisfies in the Fokker-Planck equation, then it is the density function of random process $X(t)$ which satisfy in the following stochastic differential equation

$$
d X=a(t, X) d t+b(t, X) d W .
$$

Obviously, if in (1.1), drift and diffusion coefficients are independent of $t$ (these conditions valid for stationary distributions), then the corresponding stochastic differential equation and the Fokker-Plank equation will be;

$$
\frac{d(a(x) f(x))}{d x}=\frac{1}{2} \frac{d^{2}\left(b^{2}(x) \cdot f(x)\right)}{d x^{2}}, d X=a(X) d t+b(X) d W .
$$

Langtangen ([15]) shows that the analytical solution of Fokker-Planck equation in this case is;

$$
f(x)=\frac{C_{0}}{b^{2}(x)} \exp \left[2 \int_{x_{0}}^{x} \frac{a(t)}{b^{2}(t)} d t\right],
$$

where $C_{0}$ is a normalizing constant. Also, he derived "when $b^{2}(x)=2 \pi S_{0}$, where $S_{0}$ is a constant the SDE in (1.2) can be interpreted as an equation for the velocity $X$ of a particle subjected to a viscous force $a(x)$ and a white noise excitation with constant spectral density equal to $S_{0}$. Such models are relevant for studying one-dimensional Brownian motion in gases and liquids. By allowing $b^{2}(x)$ to vary with $x$ the Fokker-Planck equation also has important applications in the description of energy envelops of stochastic oscillators". Here, SDE of some continuous distributions are obtained and then, their analytical or numerical solutions are calculated.

\section{Numerical methods of SDE and error estimation}

Consider $Y=\left\{Y(t), t_{0} \leq t \leq T\right\}$ satisfy in stochastic differential equation $d Y=a(Y) d t+b(Y) d W$. Let $X=\left\{X(t), t_{0} \leq t \leq T\right\}$ be a stochastic process that obtain from numerical method and

$$
X_{n+1}=X_{n}+\left(\tau_{n+1}-\tau_{n}\right) a\left(X_{n}\right)+b\left(X_{n}\right) \cdot\left(W_{\tau_{n+1}}-W_{\tau_{n}}\right),
$$

which known as the Euler-Maruyama (Kloeden et al. [13]) method with time approximation $t_{0}<\tau_{1}<\tau_{2}<\cdots<\tau_{N}=T$ on the time interval $[0, T]$, where this interval discrete construction uniformly, i.e., $\tau_{n}=t_{0}+n \delta$ and $\delta=\frac{T-t_{0}}{N}$. When the drift coefficient vanishes then (2.1) becomes the Euler method for ordinary differential equations. The main problem is generation of stochastic increments $\Delta W_{n}=W_{\tau_{n+1}}-W_{\tau_{n}}$ for $n=0,1,2, \ldots, N-1$. Here, these increments are generated with randn command in Matlab software, because $\frac{\Delta W_{n}}{\sqrt{\Delta_{n}}} \sim \operatorname{Normal}(0,1)$. If the exact solution is known, the absolute error may be calculated that means $\varepsilon=E\left|Y(T)-X_{T}\right|$, where $X_{T}$ and $Y(T)$ are the values 
of $X$ and $Y$ at time $T$. Assume $N$ different sample paths of Wiener process and corresponding the Euler approximations of them on the same sample paths of Wiener process are simulated. Let $Y(T, k)$ and $X_{T, k}$ be the values of Wiener process and the Euler approximation at time $T$ and on $k$-th simulation path, respectively. Now, the error is

$$
\hat{\varepsilon}=\frac{1}{N} \sum_{k=1}^{N}\left|Y(T, k)-X_{T, k}\right| .
$$

For example, consider the Ito stochastic differential equation

$$
\left\{\begin{aligned}
d Y(t) & =\mu Y(t) d t+\sigma Y(t) d W \\
Y(0) & =Y_{0}
\end{aligned}\right.
$$

where $\mu, \sigma>0$. The exact solution is;

$$
Y(t)=Y_{0} \exp \left[\left(\mu-\frac{1}{2} \sigma^{2}\right) t+\sigma \cdot W(t)\right] .
$$

For simulation on a sample path of the Euler approximation, with using initial value $X_{0}=Y_{0}$ and (2.1), $X_{n}$ are obtained recursively. Now, the exact and numerical solutions on same path of Wiener process at time $T$ are comparable, so

$$
Y\left(\tau_{n}\right)=Y_{0} \exp \left[\left(\mu-\frac{1}{2} \sigma^{2}\right) \tau_{n}+\sigma \cdot \sum_{i=1}^{n} \Delta W_{i-1}\right]
$$

for $N=25$ path of $Y(t)$ satisfy in (2.3) with $Y_{0}=1, \mu=2$ and $\sigma=1$.

Table 1: Absolute errors $\hat{\varepsilon}_{1}, \hat{\varepsilon}_{2}$

\begin{tabular}{|c||c|c|c|c|}
\hline$\delta$ & $2^{-4}$ & $2^{-5}$ & $2^{-6}$ & $2^{-7}$ \\
\hline$\hat{\varepsilon}_{1}$ & 0.8139 & 0.6315 & 0.4374 & 0.3266 \\
\hline$\hat{\varepsilon}_{2}$ & 1.2553 & 1.1088 & 0.7201 & 0.4603 \\
\hline
\end{tabular}

Table 1, shows the error according to (2.2) with step length $\delta=2^{-4}, 2^{-5}, 2^{-6}$, $2^{-7}$ and initial seed values $94\left(\hat{\varepsilon_{1}}\right)$ and $194\left(\hat{\varepsilon_{2}}\right)$ for generating random number in time interval $[0,1]$. However these approximations are random variables and have different values in a batch, but for large $N$ and applying the Central Limit Theorem, we consider the error $\hat{\varepsilon}$ as an normal random variable, so $\sigma_{\varepsilon}^{2}$ be estimated as follows and may calculate a confidence interval for absolute error $\varepsilon$. First simulated values are sorted in $M$ batches of $N$ paths. Assume $X_{T, k, j}$ be the Euler approximation on $k$-th path in $j$-th batch at time $T$ and $Y(T, k, j)$ is its corresponding process. The mean error is

$$
\hat{\varepsilon_{j}}=\frac{k-1}{N} \sum_{k=1}^{N}\left|Y(T, k, j)-X_{T, k, j}\right|, j=1,2, \ldots, M .
$$

Now, we use the student $T$-distribution for calculating the confidence interval for sums of normal variables because variance is unknown. The mean and 

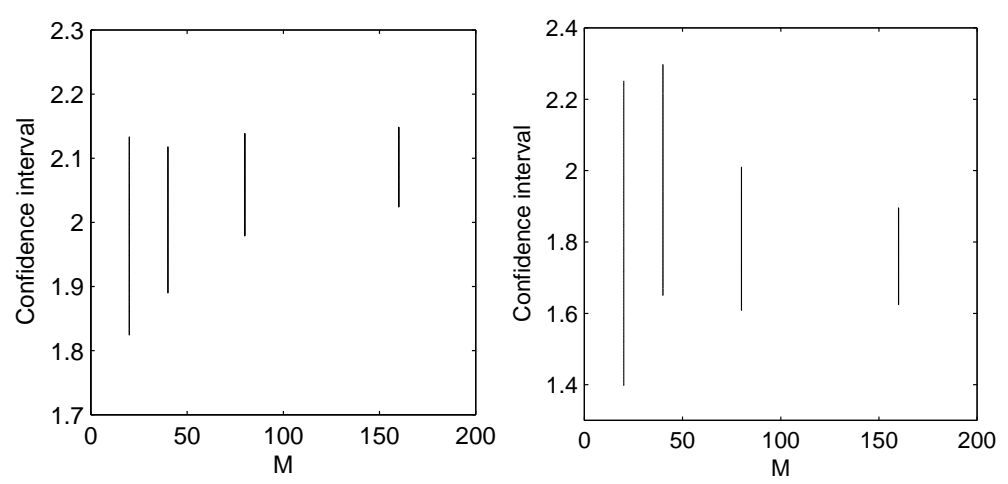

FiguRe 1. Confidence interval for absolute error corresponding to each batch for initial seed values 94(left) and 194(right).

variance of error for each batch are

$$
\hat{\varepsilon}=\frac{1}{M} \sum_{j=1}^{M} \hat{\varepsilon_{j}}, \hat{\sigma}_{\varepsilon}^{2}=\frac{1}{M-1} \sum_{j=1}^{M}\left(\hat{\varepsilon_{j}}-\hat{\varepsilon}\right)^{2} .
$$

Therefore, a confidence interval $(1-\alpha)$ percent for $\varepsilon$ is $(\hat{\varepsilon}-\Delta \hat{\varepsilon}, \hat{\varepsilon}+\Delta \hat{\varepsilon})$ where $\Delta \hat{\varepsilon}=t_{\frac{\alpha}{2}}(M-1) \frac{\hat{\sigma}_{\varepsilon}}{\sqrt{M}}$. Assume $M=20, \alpha=0.1$, then $t_{0.05}(19)=2.09$. For $N=100$ the Wiener process path with $Y_{0}=1, \mu=2, \sigma=1$ and the Euler approximation with step length $\delta=2^{-4}$ correspond to the same sample paths of the Wiener process on $[0,1]$, the confidence interval 90 percent is calculated for $\varepsilon$. These procedure are repeated for $M=40,80,160$ and the results are shown in Figure 1. We observe that increasing batches, decrease confidence interval width.

\section{Stochastic differential equation for continuous distributions}

In this section, with using Fokker-Planck equation, for stochastic process $\{Y(t)\}$, stochastic differential equation and corresponding equation for density function are determined and then equations with nonlinear coefficients are approximated with some methods like Euler-Maruyama and equations with linear coefficients may be solved numerically and analytically. Here, we only describe detail of the method for exponential and normal distributions and method for other distributions are similar. Assume $Y$ is a continuous random variable with corresponding stochastic differential equation $d Y=a(Y) d t+b(Y) d W$. It is clear that, different choices of $a(x)$ give several common probability densities. Next, using conditions for diffusion process and also experimented methods, consider drift coefficient $a(Y)$ in form of $-\alpha(Y-E Y)$, where $\alpha$ is a positive constant real number. 


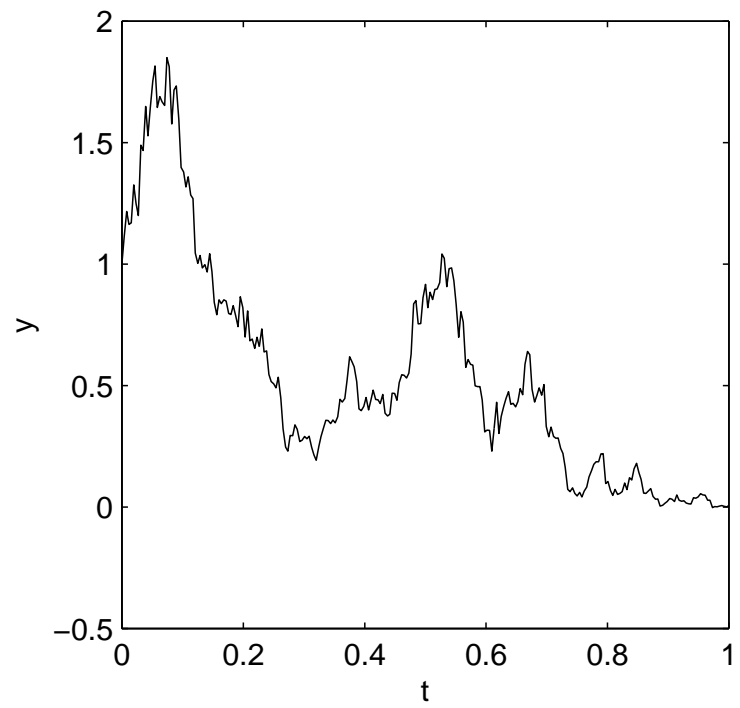

FiguRE 2. Numerical solution of SDE corresponds to the exponential distribution with parameters $\alpha=\lambda=2$.

\subsection{Exponential distribution}

Let $Y \sim \exp (\lambda)$ with density function $f(y)=\lambda \exp [-\lambda y], y \geq 0$, and drift coefficient $a(y)=\left(-\alpha\left(y-\frac{1}{\lambda}\right)\right)$. From the Fokker-Plank equation be deduced:

$$
\frac{d}{d y}\left[-\alpha\left(y-\frac{1}{\lambda}\right) \cdot \lambda \exp (-\lambda y)\right]=\frac{1}{2} \frac{d^{2}}{d y^{2}}\left[b^{2}(y) \cdot \lambda \exp (-\lambda y)\right]
$$

Assume $g(y)=b^{2}(y)$ and from solution of the above differential equation, we have

$$
g(y)=\left(c_{1}+c_{2}\right) e^{\lambda y}+\frac{2 \alpha}{\lambda} y .
$$

The diffusion coefficient $b(y)$ should satisfy to the Lipschitz condition and linear growth bounded, then $c_{1}=c_{2}=0$ and $b(y)=\sqrt{\frac{2 \alpha}{\lambda}} y$. Therefore, the following stochastic differential equation will be obtained

$$
d y=-\alpha\left(y-\frac{1}{\lambda}\right) d t+\sqrt{\frac{2 \alpha}{\lambda}} y d W(t) .
$$

Figure 2 shows numerical approximation of the above SDE by the EulerMaruyama method with $\lambda=\alpha=2$. 


\subsection{Normal distribution}

Let $Y \sim N\left(\mu, \sigma^{2}\right)$ with drift coefficient $a(y)=-\alpha(y-\mu)$. From the FokkerPlanck equation, we have

$$
\frac{d}{d y}\left[-\alpha(y-\mu) \frac{1}{\sqrt{2 \pi} \sigma} \exp \left[-\frac{(y-\mu)^{2}}{2 \sigma^{2}}\right]\right]=\frac{1}{2} \frac{d^{2}}{d y^{2}}\left[b^{2}(y) \frac{1}{\sqrt{2 \pi} \sigma} \exp \left[-\frac{(y-\mu)^{2}}{2 \sigma^{2}}\right]\right]
$$

with solving the ordinary differential equation, we obtain $b(y)=\sqrt{2 \alpha} \sigma$. Hence stochastic differential equation is:
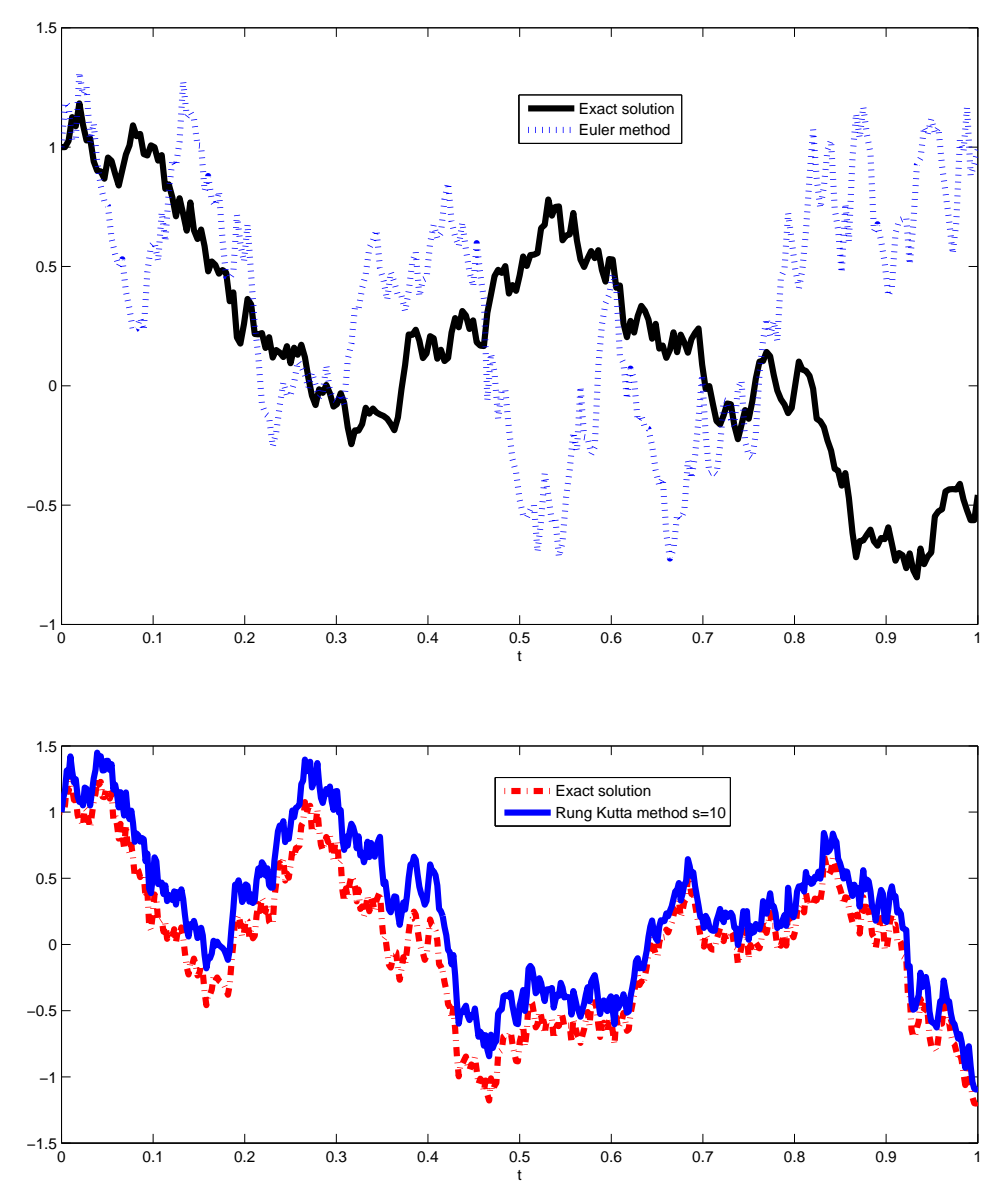

FiguRE 3. Numerical and exact solutions of (1.3) with the Euler-Maruyama method(top) and 10 stages explicit RungeKutta method(down) corresponding to the normal distribution with parameters $Y_{0}=1, \alpha=2, \mu=0, \sigma=1$. 


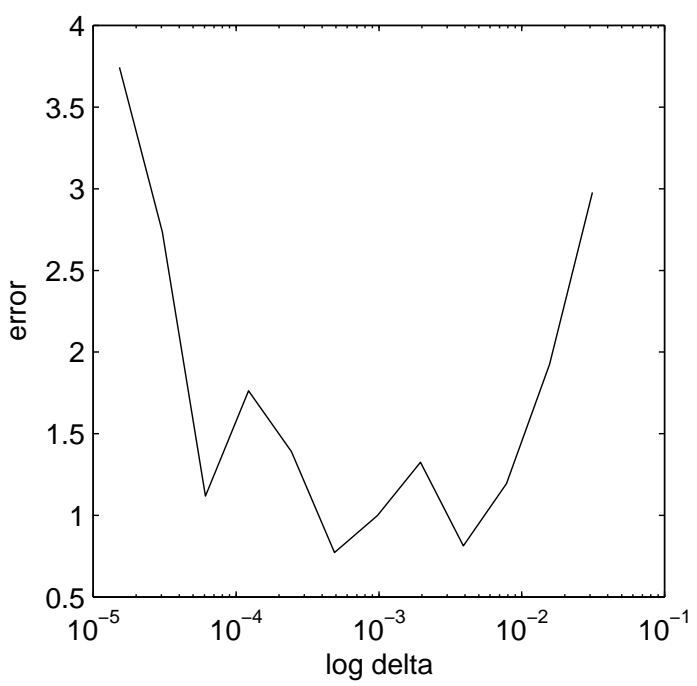

Figure 4. Absolute error with respected to logarithm of long walks $2^{-6}, 2^{-7}, \ldots, 2^{-16}$.

$$
d Y=-\alpha(y-\mu) d t+\sqrt{2 \alpha} \sigma d W(t) .
$$

The coefficient of the above SDE is linear, so the exact solution will be (Kloeden and Platen, [13])

$$
Y(t)=\exp (-t \alpha)\left(Y_{0}-\mu(1-\exp (-t \alpha))+\sqrt{2 \alpha} \sigma \int_{0}^{t} \exp (s \alpha) d W(s) .\right.
$$

The numerical approximation with the Euler-Maruyama method and the solution with parameters $\alpha=2, \mu=0, \sigma=1$ and initial seed value 13 will be presented in Figure 3. The error for $N=2^{9}$ on 35 path is 0.445 . For 25 paths of process $Y(t)$ that satisfy in (1.3) with $\alpha=2, Y_{0}=1, \mu=0, \sigma=1$.

Table 2: Absolute error $\varepsilon$

\begin{tabular}{|c||c|c|c|c|}
\hline$\delta$ & $2^{-5}$ & $2^{-6}$ & $2^{-7}$ & $2^{-8}$ \\
\hline$\varepsilon$ & 2.9775 & 1.9241 & 1.1935 & 0.8132 \\
\hline
\end{tabular}

Table 2 shows the error of $(2.2)$ with step length $\delta=2^{-5}, 2^{-6}, 2^{-7}, 2^{-8}$ and initial seed value 94 for generating random number on time interval $[0,1]$. The graph of absolute error vice versa logarithm of the length $2^{-5}, 2^{-6}, \ldots, 2^{-16}$ be shown in Figure 4.

\subsection{Other distributions}

With similar method and by the Fokker-Plank theorem, we obtain the Fokker-Plank and the stochastic differential equations for the following distributions and be presented in Table 4 . 
- Double exponential distribution with parameters $\eta$ and $\theta$.

- Pareto distribution with parameters $k$ and $\theta$

- Gamma distribution with parameters $k$ and $\theta$

- Beta distribution with parameters $a$ and $b$

- Uniform distribution with parameters $a$ and $b$

\section{Runge-Kutta method for solving SDE}

One of approximation method for SDE $d Y(t)=f(Y(t)) d t+g(Y(t)) d W(t)$ is Runge-Kutta method where be defined as follows;

$$
\left\{\begin{aligned}
Y_{h}= & Y_{0}+\sum_{j=1}^{s} c_{j} Y_{j}, \\
Y_{i}= & h_{i} a_{i} f\left(Y_{0}+\sum_{j=1}^{s} \alpha_{i j} Y_{j}\right)+h_{i} f^{(1)}\left(Y_{0}\right) \sum_{j=1}^{s} \gamma_{i j} Y_{j} \\
& +w_{i} b_{i} g\left(Y_{0}+\sum_{j=1}^{s} \beta_{i j} Y_{j}\right)+w_{i} g^{(1)}\left(Y_{0}\right) \sum_{j=1}^{s} \delta_{i j} Y_{j},
\end{aligned}\right.
$$

diffusion coefficient of SDE has normal distribution independent of $y$ and so the solution of above differential equation equivalence to the solution of corresponding Itô-differential equation. According to the order conditions (Komori, et al., [14]), coefficients $a_{i}, b_{i}, c_{i}$ and matrices $\alpha, \beta, \gamma, \delta$ for 10 stages explicit Runge-Kutta method are obtained (Table 3), therefore

$$
\begin{aligned}
Y_{h}= & Y_{0}+\frac{1}{2} h \cdot f(\bar{y})+\frac{1}{2} h \cdot f\left(Y_{0}\right)+\frac{1}{4} h \cdot g\left(Y_{0}\right) g^{(1)}\left(Y_{0}\right)+\frac{1}{2} \Delta W \cdot g\left(Y_{0}\right) \\
& +\frac{\sqrt{h}}{24}\left[8\left\{g\left(\bar{y}+\frac{1}{2} \sqrt{h} g(\bar{y})\right)-g\left(\bar{y}-\frac{1}{2} \sqrt{h} g(\bar{y})\right)\right\}\right. \\
& \left.-\left\{g(\bar{y}+\sqrt{h} g(\bar{y}))-g\left(\bar{y}-\frac{1}{2} \sqrt{h} g(\bar{y})\right)\right\}\right] \\
& +\frac{1}{4} g\left(\bar{y}^{+}\right)\left\{\Delta W+\left((\Delta W)^{2}-h\right) h^{\frac{1}{2}}\right\}+\frac{1}{4} g\left(\bar{y}^{-}\right)\left\{\Delta W+\left((\Delta W)^{2}-h\right) h^{\frac{1}{2}}\right\},
\end{aligned}
$$

Table 3: Parameters of explicit Runge-Kutta method 10 stage

\begin{tabular}{|c||c|}
\hline Parameters & Conditions \\
\hline$a_{i}, \alpha_{i j}, \gamma_{i j}, \delta_{i j}$ & $a_{3}=a_{5}=1, \alpha_{51}=\alpha_{32}=1, \delta_{32}=0.5$ and 0 otherwise \\
\hline$b_{i}$ & $b_{3}=0, b_{5}=-\frac{1}{12}$ and 1 otherwise \\
\hline$c_{i}$ & $c_{1}=c_{3}=c_{5}=\frac{1}{2}, c_{6}=\frac{1}{24}, c_{7}=\frac{1}{3}, c_{8}=-c_{7}, c_{9}=c_{10}=\frac{1}{4}$ and 0 otherwise \\
\hline$w_{i}$ & $w_{1}=\Delta W, w_{2}=w_{3}=w_{4}=w_{5}=w_{6}=w_{7}=w_{8}=\sqrt{h}$, \\
& $w_{9}=\Delta W+\left((\Delta W)^{2}-h\right) h^{-\frac{1}{2}}, w_{10}=\Delta W-\left((\Delta W)^{2}-h\right) h^{-\frac{1}{2}}$ \\
\hline$\beta_{i j}$ & $\beta_{41}=\beta_{43}=\beta_{51}=\beta_{53}=\beta_{54}=\beta_{61}=\beta_{63}=\beta_{71}=\beta_{73}=\beta_{81}=\beta_{83}=\beta_{92}=\beta_{93}=\beta_{10,3}=1$, \\
& $\beta_{64}=\beta_{10,2}=-1, \beta_{74}=\frac{1}{2}, \beta_{84}=-\frac{1}{2}$ and 0 otherwise \\
\hline
\end{tabular}

where $\Delta W$ is a random variable of normal distribution with mean zero and variance $h$ and

$$
\begin{aligned}
& \bar{y}=Y_{0}+h\left(f\left(Y_{0}\right)+\frac{1}{2} g\left(Y_{0}\right) g^{(1)}\left(Y_{0}\right)\right)+g\left(Y_{0}\right) \Delta W, \\
& \bar{y}^{ \pm}=Y_{0}+h\left(f\left(Y_{0}\right)+\frac{1}{2} g\left(Y_{0}\right) g^{(1)}\left(Y_{0}\right)\right) \pm g\left(Y_{0}\right) \sqrt{h} .
\end{aligned}
$$

The graph of exact and numerical solutions with 10 stages explicit RungeKutta method for SDE corresponding the normal distribution with parameters $Y_{0}=1, \alpha=2, \mu=0, \sigma=1$ and initial seed value 13 for generating random 
number over $[0,1]$ and $h=2^{-9}$ and 25 paths is 0.3846 , is shown in Figure 3 (below).

Table 4: SDE, Fokker-Plank and corresponding distribution

\begin{tabular}{|c|c|c|}
\hline Distribution & Fokker-Plank equation & SDE \\
\hline $\exp (\lambda)$ & $\begin{array}{c}\frac{d}{d y}\left[-\alpha\left(y-\frac{1}{\lambda}\right) \cdot \lambda e^{-\lambda y}\right]= \\
\frac{d^{2}}{2 d y^{2}}\left[b^{2}(y) \cdot \lambda e^{-\lambda y}\right]\end{array}$ & $d y=-\alpha\left(y-\frac{1}{\lambda}\right) d t+\sqrt{\frac{2 \alpha}{\lambda}} d W(t)$ \\
\hline$N\left(\mu, \sigma^{2}\right)$ & $\begin{array}{c}\frac{d}{d y}\left[-\alpha(y-\mu) \frac{1}{\sqrt{2 \pi} \sigma} e^{-\frac{(y-\mu)^{2}}{2 \sigma^{2}}}\right]= \\
\frac{d^{2}}{2 d y^{2}}\left[b^{2}(y) \cdot \frac{1}{\sqrt{2 \pi} \sigma} e^{-\frac{(y-\mu)^{2}}{2 \sigma^{2}}}\right]\end{array}$ & $d y=-\alpha(y-\mu) d t+\sqrt{2 \alpha} \sigma d W(t)$ \\
\hline DubleExp $(\eta, \theta)$ & $\begin{array}{c}\frac{d}{d y}\left[-\alpha(y-\eta-\theta) \cdot \frac{1}{\theta} e^{-\frac{y-\eta}{\theta}}\right]= \\
\frac{d^{2}}{2 d y^{2}}\left[b^{2}(y) \cdot \frac{1}{\theta} e^{-\frac{y-\eta}{\theta}}\right]\end{array}$ & $d y=-\alpha(y-\eta-\theta) d t+\sqrt{2 \alpha \theta(y-\eta)} d W(t)$ \\
\hline $\operatorname{Gamma}(k, \theta)$ & $\begin{array}{c}\frac{d}{d y}\left[-\alpha(y-k \theta) \cdot \frac{1}{\theta^{k} \Gamma(k)} y^{k-1} e^{-\frac{y}{\theta}}\right]= \\
\frac{d^{2}}{2 d y^{2}}\left[b(y) \cdot \frac{1}{\theta^{k} \Gamma(k)} y^{k-1} e^{-\frac{y}{\theta}}\right]\end{array}$ & $d y=-\alpha(y-k \theta) d t+\sqrt{2 \alpha \theta y} d W(t)$ \\
\hline $\operatorname{Pareto}(k, \theta)$ & $\begin{array}{c}\frac{d}{d y}\left[-\alpha\left(y-\frac{\theta}{k-1}\right) \cdot \frac{k}{\theta}\left(1+\frac{y}{\theta}\right)^{-k-1}\right]= \\
\frac{d^{2}}{d y^{2}}\left[b^{2}(y) \cdot \frac{k}{\theta}\left(1+\frac{y}{\theta}\right)^{-k-1}\right]\end{array}$ & $d y=-\alpha\left(y-\frac{\theta}{k-1}\right) d t+\sqrt{\frac{2 \alpha y(y+\theta)}{k-1}} d W(t)$ \\
\hline $\operatorname{Betta}(a, b)$ & $\begin{array}{c}\frac{d}{d y}\left[-\alpha\left(y-\frac{a}{a+b}\right) \cdot \frac{\Gamma(a+b)}{\Gamma(a) \Gamma(b)} y^{a-1}(1-y)^{b-1}\right] \\
\frac{d^{2}}{d y^{2}}\left[b^{2}(y) \cdot \frac{\Gamma(a+b)}{\Gamma(a) \Gamma(b)} y^{a-1}(1-y)^{b-1}\right]\end{array}$ & $d y=-\alpha\left(y-\frac{a}{a+b}\right) d t+\sqrt{\frac{2 \alpha y(1-y)}{a+b}} \cdot d W(t)$ \\
\hline$U(a, b)$ & $\begin{array}{c}\frac{d}{d y}\left[-\alpha\left(y-\frac{a+b}{2}\right) \cdot \frac{1}{b-a}\right]= \\
\frac{d^{2}}{2 d y^{2}}\left[b(y) \cdot \frac{1}{b-a}\right]\end{array}$ & $d y=-\alpha\left(y-\frac{a+b}{2}\right) d t+\sqrt{\alpha(y-a)(b-y)} d W(t)$ \\
\hline
\end{tabular}

\section{Conclusions}

Among continuous distributions, SDE corresponding normal distribution is an equation with linear coefficients and may calculate the exact solution. In the pervious section, the absolute error for different stage and estimation of confidence interval have shown with different batch length. The easiest numerical approximation of differential equation is the Euler method. As in Figure 3 is shown, numerical and simulation exact solutions may have not same behavior with Euler method, but 10 stages explicit Runge-Kutta method have higher weak convergency order with respect to the Euler, (Kloeden and Platen, [13] and Komori, et al., [14]) and numerical solution have higher accuracy. Therefore, if we choose the normal distribution as a test problem, the numerical solution for other distributions with this Runge-Kutta method have good accuracy.

Acknowledgement. The authors would like to thank the referees for their careful reading of the manuscript and for valuable suggestions which improved the presentation of the paper. This research was supported by a grant from Ferdowsi University of Mashhad (No. MS89171AMI).

\section{References}

[1] L. Ambrosio, G. Savaré, and L. Zambotti, Existence and stability for Fokker-Planck equations with log-concave reference measure, Probab. Theory Related Fields $\mathbf{1 4 5}$ (2009), no. 3-4, 517-564.

[2] E. Barkai, Fractional Fokker-Planck equation, solution, and application, Phys. Rev. E 63 (2001), 046118. 
[3] E. Buckwar, Introduction to the numerical analysis of stochastic delay differential equations, J. Comput. Appl. Math. 125 (2000), no. 1-2, 297-307.

[4] K. Burrage, P. M. Burrage, and T. Tian, Numerical methods for strong solutions of stochastic differential equations: an overview, Proc. R. Soc. Lond. Ser. A Math. Phys. Eng. Sci. 460 (2004), no. 2041, 373-402.

[5] K. Burrage and P. M. Burrage, Numerical methods for stochastic differential equations with applications, advanced computational modeling center, Unversity of Queensland, Australia, 2002.

[6] M. Careltti, Numerical solution of stochastic differential problems in the biosciences, J. Comput. Appl. Math. 185 (2006), no. 2, 422-440.

[7] J. B. Chen and J. Li, A note on the principle of preservation of probability and probability density evolution equation, Probab. Eng. Mech. Vol. 24 (2009), 51-59.

[8] S.-N. Chow and H.-M. Zhou, An analysis of phase noise and Fokker-Planck equations, J. Differential Equations 234 (2007), no. 2, 391-411.

[9] O. Ditlevsen, Invalidity of the spectral Fokker-Planck equation for Cauchy noise driven Langevin equation, Probab. Eng. Mech. 19 (2004), no. 4, 385-392.

[10] T. D. Frank and A. Daffertshofer, Nonlinear Fokker-Planck equations whose stationary solutions make entropy-like functionals stationary, Phys. A 272 (1999), no. 3-4, 497508 .

[11] T. D. Frank, A note on the Markov property of stochastic processes described by nonlinear Fokker-Planck equations, Phys. A 320 (2003), no. 1-4, 204-210.

[12] D. Kim and D. Stanescu, Low-storage Runge-Kutta methods for stochastic differential equations, Appl. Numer. Math. 58 (2008), no. 10, 1479-1502.

[13] P. E. Kloeden and E. Platen, Numerical Solution of Stochastic Differential Equations, Springer-Verlage, Berlin, 1992.

[14] Y. Komori, T. Mistsui, and H. Sugiura, Rooted tree analysis of the order continuous of row-type scheme for stochastic differential equation, BIT, 37 (1997), no. 1, 43-66.

[15] H. P. Langtangen, A general numerical solution method for Fokker-Planck equations with applications to structural relibaility, Probab. Eng. Mech. 6 (1991), no. 1, 33-48.

[16] X. Mao and S. Sabanis, Numerical solutions of stochastic differential delay equations under local Lipschitz condition, J. Comput. Appl. Math. 151 (2003), no. 1, 215-227.

[17] X. Mao, C. Yuan, and G. Yin, Numerical method for stationary distribution of stochastic differential equations with Markovian switching, J. Comput. Appl. Math. 174 (2005), no. $1,1-27$.

[18] H. C. Ottinger, Stochastic Processes in Polymeric Fluids, Springer Verlag, Berlin, 1999.

[19] M. Di Paola and A. Sofi, Approximate solution of the Fokker-Planck-Kolmogorov equation, Probab. Eng. Mech. 17 (2002), 369-384.

[20] S. Primak, V. Kontorovich, and V. Lyandres, Stochastic Methods and Their Applications to Communications, John Wiley \& Sons Ltd, 2004.

[21] H. Risken, The Fokker-Planck Equation, Second Edition. Springer, 1996.

[22] Jr. M. S. Torres and J. M. A. Figueiredo, Probability amplitude structure of Fokker-Plank equation, Phys. A 329 (2003), no. 1-2, 68-80.

[23] A. Tocino and R. Ardanuy, Runge-Kutta methods for numerical solution of stochastic differential equations, J. Comput. Appl. Math. 138 (2002), no. 2, 219-241.

MOHAMmad Amini

Department of Statistics

Ordered and Spatial Data Center of Excellence(OSDCE)

FERDOWSI UNIVERSITY OF MASHHAD

MASHHAD 91775-1159, IRAN

E-mail address: m-amini@um.ac.ir 
Ali Reza Soheili

Department of Applied Mathematics

The CENTER OF EXCELLENCE IN MODELING

AND COMPUTATIONS IN LINEAR AND NONLINEAR SYSTEMS(CRMCS)

FERDOWSI UNIVERSITY OF MASHHAD

MASHHAD 91775-1159, IRAN

E-mail address: soheili@um.ac.ir

Mahdi Allahdadi

Department of Mathematics

UNIVERSity OF Sistan AND BALUCHESTAN

ZAHEDAN 98135-674, IRAN

E-mail address: mehdi-1486@yahoo.com 